\title{
Nazopharyngeal pneumococcal carriage in healthy Turkish children after 13-valent conjugated pneumococcal vaccination
}

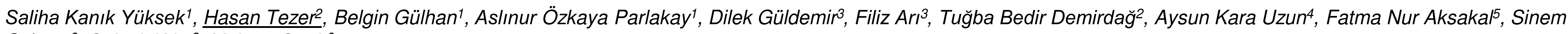
Solmaž, Selçuk Kılıç3, Meltem Çırak'

${ }^{1}$ Ankara Hematology Oncology Children's Training and Research Hospital, Pediatric Infectious Diseases Department, Ankara, Turkey

${ }^{2}$ Gazi University Medical Faculty, Pediatric Infectious Diseases Department, Ankara, Turkey

${ }^{3}$ Ministry of Health, Public Health Agency of Turkey, the National Reference Laboratory for Molecular Microbiology, Ankara

${ }^{4}$ Ankara Hematology Oncology Children's Training and Research Hospital, Pediatric Diseases Department, Ankara, Turkey

${ }^{5}$ Gazi University Medical Faculty, Public Health Department, Ankara, Turkey

${ }^{6}$ Gazi University Medical Faculty, Medical Microbiology Department, Ankara, Turkey

Background: The source of pneumococcal infections causing significant morbidity and mortality is mainly asymptomatic carriers. Along with the widespread use of conjugated pneumococcal vaccines all over the world, it is expected that the frequency of invasive pneumococcal disease will decrease in parallel with the decrease in the proportion of carriers. In Turkey, pneumococcal 7-valent conjugate vaccine (PCV7) in 2008 and PCV13 in 2011 were introduced to the national immunization program. The purpose of this study is to investigate the effects of pneumococcal conjugate vaccine (PCV13) on nasopharyngeal carriage rates of Streptococcus pneumoniae, and to determination the pneumococcal serotype distribution in healthy Turkish children.

Methods: The study was conducted on 500 healthy Turkish children between 0 month and 13 years of age. Samples were taken as nasopharyngeal swab samples from April to November 2014 from children who do not have a history of infection and antibiotic use within the last two weeks. Received samples were delivered to the laboratory in the preventive carrier medium. Samples were incubated on $5 \%$ sheep blood agar at $37{ }^{\circ} \mathrm{C}$ with $5-10 \% \mathrm{CO}^{2}$ for $18-24$ hours. Following the incubation, the optokin susceptibility test was performed to confirm that the colonies obtained were $S$. pneumoniae. Capsular serotyping by molecular method was performed on the isolates which were confirmed to be pneumococci.
In this method, the presence of $S$. pneumoniae DNA was first researched by sensitive real-time PCR based on lytA gene amplification. Subsequently, the isolates were examined by multiplex PCR for the presence of pneumococcal-specific cpsA gene and vaccine serotype content (PCV13). Strains that were found to be non-vaccine serotype by this method were further typed by a different multiplex PCR kit.

Results: Of the 500 children who participated in the study, $278(55.6 \%)$ were male and 221 (44.4\%) female. The mean age of the children was $55.97 \pm 50.23(0-156)$ months. Number of children under the age of $<2$ (0-23 months, I. group) was 195 (39\%) aged 2-5 (24-59 months, II. group) years 95 (19\%) and aged $\geq 5$ (60 months and over, III. group) was 210 (42\%). Nasopharyngeal pneumococcal carriage (NPC) was detected in $49(9.8 \%)$ of the 500 healthy children. Of the isolates, $26(53 \%)$ were in PCV13 vaccine strains. Distribution of vaccine strains was as follows; serotype $318.3 \%$ (9/49), serotype $19 \mathrm{~F} 14.2 \%$ (7/49), serotype 6A / B $8.2 \%$ (4/49), serotype 18C 4\% (2/49), serotype 19A $2 \%$ ), and serotype 9V\% 2 $(1 / 49)$. Distribution of non-vaccine strains is; serotype 11A (4/49), serotype 15B (4/49), serotype 23A (3/49), serotype 9L / N (1/49), serotype $15 \mathrm{~A}(1 / 49)$, serotype $16 \mathrm{~F}(1 / 49)$ serotype 31 (1/49), serotype 35B (1/49), and serotype $35 \mathrm{~F}(1 / 49)$. Type of the six isolates $(12.2 \%)$ was not identified.
In group I NPC was found as 11.8\% ( $\mathrm{n}=$ $23 / 195)$, in group II $8.4 \%(n=8 / 95)$ and in the group III $8.6 \%(n=18 / 210)$, there was no significant difference between the groups $(p=$ $0.487)$. Of the children, $42.2 \%(n=211)$ were unvaccinated with a conjugate pneumococcal vaccine (7 or 13 -valent), $25.6 \%(n=128)$ were incomplete vaccinated and $32.2 \%(n=$ 161) were full vaccinated. Among all age groups, it was found that incomplete vaccination with 13-valent vaccine in I. group found to increase NPC $(p=0.001)$. The serotypes most frequently detected by the groups were; in I. group serotype and nontyped; in II. group serotype 3, 19F, 6A / B $11 \mathrm{~A}, 35 \mathrm{~F}, 16 \mathrm{~F}$ and non-typed ; in III. group serotype 3,15B and 19F. Vaccination status of the carriers is shown on chart $(p=0.006)$.
Conclusions: Compared with nasopharyngeal carriage studies in Turkey conducted in the pre- and post-vaccine (PCV7) era, a significant decrease in carriage rates was detected in three years after the introduction of PCV13 (from 21.9\% to 9.8\%). However, the nasopharyngeal carriage is particularly high in PCV13 strains interestingly. The finding of $19 \mathrm{~F}$ as the second most frequently detected serotype in our study is significant in that it shows that the effect of PCV13 on serotype 19F is not much different from PCV7. Since pneumococcal surveillance data varies from country to country, it is important for each country to identify and monitor its national pneumococcal surveillance.

\section{Vaccination status of the carriers}

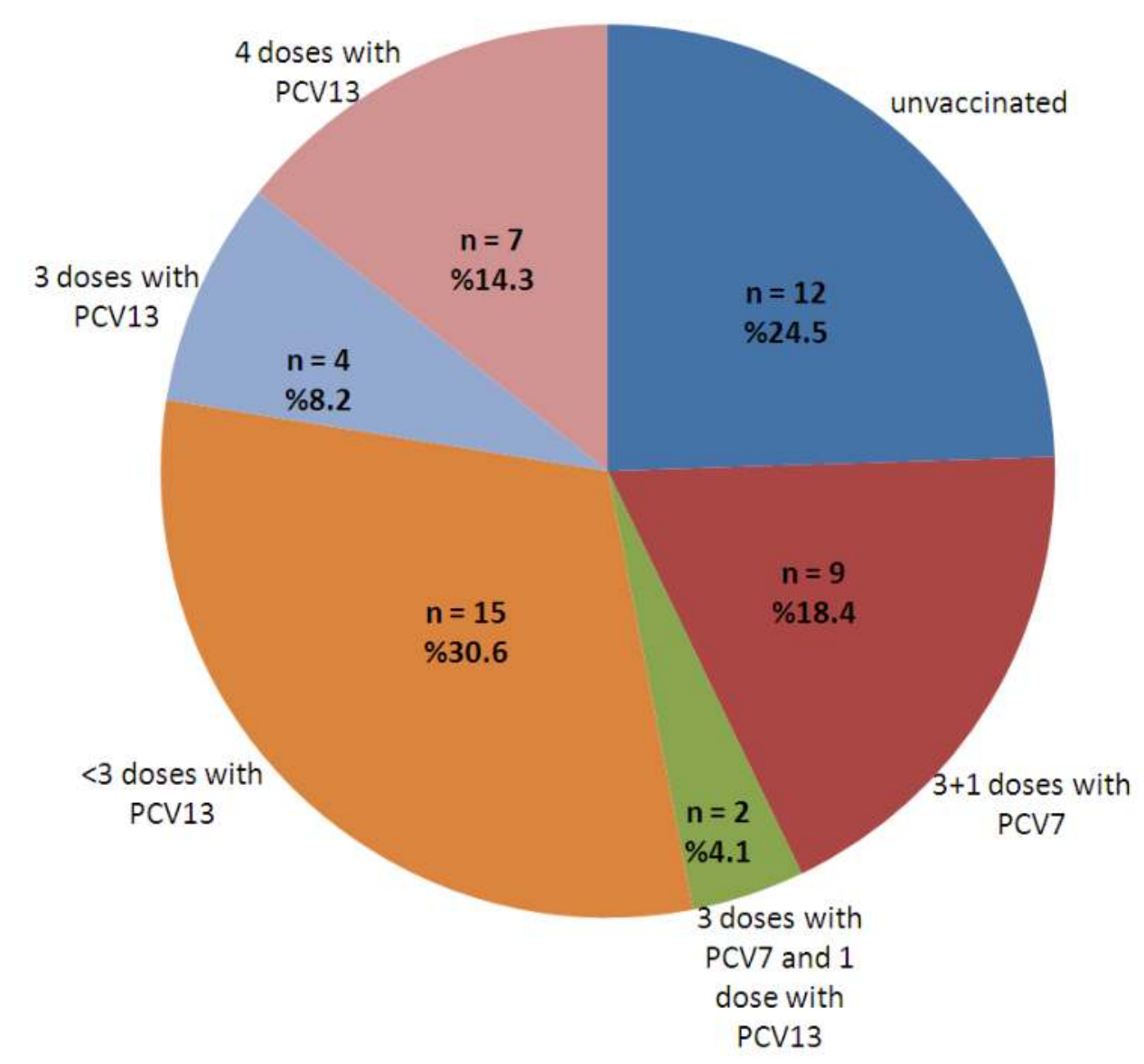

